\title{
Validation of WHO-IMNCI algorithm for jaundice in 0-2 months aged infants
}

\author{
Bansal R. ${ }^{1}$, Sharma R.K. ${ }^{2}$ \\ ${ }^{1}$ Dr. Rishi Bansal, Assistant Professor, Department of Paediatrics, FH Medical College, Tundla, ${ }^{2}$ Dr. Ram Kshitij \\ Sharma, Assistant Professor, Department of Paediatrics, S.N. Medical College, Agra, Uttar Pradesh, India.
}

Corresponding Author: Dr. Ram Kshitij Sharma, Assistant Professor, Department of Paediatrics, S.N. Medical College, Agra, Uttar Pradesh, India. E-mail: ramkshitijsharma@gmail.com

\begin{abstract}
Background: For effective management of these major childhood illnesses, WHO and UNICEF have developed the "Integrated Management of Neonatal and Childhood Illness" (IMNCI) Strategy. Present study is to evaluate the utility of the WHO/UNICEF algorithm for Integrated Management of Neonatal and Childhood Illness (IMNCI) for jaundice up to 2 months of age. Methods: Present study is Prospective observational comparative study. Total of 150 subjects were taken from Emergency and Outpatient Department of Pediatrics. The treatment steps were identified as according to the 'Assess and Classify' module of IMNCI algorithm. All relevant investigations were performed, using appropriate methods. Blood sugar was done in all recruited children and serum bilirubin levels were done in all infants presented with jaundice. Based on this detailed clinical evaluation and relevant investigations, final diagnosis were made and therapies were given. These diagnosis and treatments were considered as the 'Gold Standard' for comparison. Results: There were 150 young infants, of whom $81(54 \%)$ were male and $69(46 \%)$ were female infants. Total of 73 infants were admitted, 12 from OPD and 61 from Emergency. 77 infants were sent home after initial management in hospital. Severe jaundice was present in 12 infants according to IMNCI and 6 infants according to Gold Standard in 0-2 months of age. The predictive utility of algorithm for the diagnosis of severe jaundice with a sensitivity of $100 \%$, specificity of $75 \%$, positive predictive value of $50 \%$ and negative predictive value of $100 \%$ in $0-2$ months of age group. Conclusion: Algorithm performed well in identifying severe jaundice with the sensitivity of $100 \%$ and specificity of $95 \%$.
\end{abstract}

Keywords: Childhood illness, Jaundice, WHO-IMNCI

\section{Introduction}

Every day, millions of parents seek health care for their sick children, taking them to hospitals, health centers, pharmacists, doctors and traditional healers. Surveys reveal that many sick children are not properly assessed and treated by these health care providers, and that their parents are poorly advised [1]. In the neonatal period, the first week is even more crucial. Three-fourth of all newborn deaths occurs during the first week of life, making it the most vulnerable period of life [2]. Any meaningful strategy to combat the childhood mortality needs to be taken into consideration for the most vulnerable period that is the early neonatal period (0-7 days) [3]. Thus, a single diagnosis for a sick child is often inappropriate because it identifies only the most apparent problem and can lead to an associated and

Manuscript received: $8^{\text {th }}$ September 2019

Reviewed: $18^{\text {th }}$ September 2019

Author Corrected: $25^{\text {th }}$ September 2019

Accepted for Publication: $30^{\text {th }}$ September 2019 potentially life-threatening problem being overlooked. For effective management of these major childhood illnesses, WHO and UNICEF have developed the "Integrated Management of Childhood Illness" (IMCI) Strategy $[4,5]$. Generic IMCI program did not initially include newborn less than one week old. There are very few published studies where IMNCI algorithm has been tested in children 7 days -2 months old $[6,7]$ within Indian subcontinent.

IMNCI is an integrated approach to child health that focuses on the well-being of the whole child. IMNCI aims to reduce death, illness and disability, and to promote improved growth and development among children under 5 years of age. IMNCI includes both preventive and curative elements that implemented by families and communities as well as by health facilities [8].

Pediatric Review: International Journal of Pediatric Research Available online at: www.medresearch.in 521|P a g e 
The present study therefore was done with the objective to evaluate the validity of IMNCI algorithm in $0-2$ months of age with special emphasis in early neonatal period.

\section{Materials and Methods}

Study Design: This study is prospective observational comparative study.

Place: It is conducted at Emergency and Outpatient Department of Pediatrics, FH Medical College and Hospital and S.N. Medical College and Hospital.

Study Duration: One Year (Jan 2017 to Dec 2017)

Sample Size: In this study total of 150 subjects were taken from Emergency and Outpatient Department of Pediatrics.

A sample size of 129 was calculated to be sufficient to detect a difference of $10 \%$ in diagnostic agreement form the gold standard with $90 \%$ power and an alpha of 0.05 .

Inclusion criteria: Any infant presented with a fresh episode of illness in the Emergency or the Outpatient Department of the Pediatrics in FHMC and SNMC were included.

Exclusion criteria: Infants attended the well-baby clinic or immunization clinic for routine visits and follow-up care were kept out of the study.

\section{Original Research Article}

Data collection: For all the children in the study, detailed history and clinical examination listed in IMNCI algorithm, in the same order. The treatment steps were identified as according to the 'Assess and Classify' module of IMNCI algorithm and was recorded in the proforma. The study subjects so selected were managed according to the protocol of treating unit under the supervision of the senior faculty. All relevant investigations were performed, using appropriate methods. Blood sugar was done in all recruited children and serum bilirubin levels were done in all infants presented with jaundice. Based on this detailed clinical evaluation and relevant investigations, final diagnosis were made and therapies were given. These diagnosis and treatments were considered as the 'Gold Standard' for comparison. The study children were either admitted or sent home after initial evaluation, depending upon the nature and severity of illness. The hospitalized subjects were followed up till the final outcome.

Feeding counseling was given to every child with low birth weight or feeding problems.

Consent: A written informed consent was taken from all the parents of babies enrolled in the study

Data analysis: The data was evaluated in the predesigned proforma, data were entered into MS Excel and analyzed using SPSS 10.01 for windows 7, chi square test and fisher's exact test, sensitivity, specificity, positive predictive value and negative predictive value.

\section{Results}

A total of 150 infants between 0-2 months who fulfilled the study criteria were studied. The baseline profile of study infants is as follows-

There were 150 young infants, of whom $81(54 \%)$ were male and 69(46\%) were female infants as shown in Table 1.

Table-1: Sex distribution of study infants (0-2 months).

\begin{tabular}{|c|c|c|}
\hline & Number & Percent (\%) \\
\hline Male & $\mathbf{8 1}$ & $\mathbf{5 4 \%}$ \\
\hline Female & $\mathbf{6 9}$ & $\mathbf{4 6 \%}$ \\
\hline Total & $\mathbf{1 5 0}$ & $\mathbf{1 0 0 \%}$ \\
\hline
\end{tabular}

Table 2 shows distribution of infants involving 51.8\% males and 48.2\% females in age group 0-7 days of age.

Table-2: Sex distribution of study infants (0-7 days).

\begin{tabular}{|c|c|c|}
\hline & Number & Percent (\%) \\
\hline Male & $\mathbf{4 2}$ & $\mathbf{5 1 . 8 \%}$ \\
\hline Female & $\mathbf{3 9}$ & $\mathbf{4 8 . 2 \%}$ \\
\hline Total & $\mathbf{8 1}$ & $\mathbf{1 0 0 \%}$ \\
\hline
\end{tabular}

Table 3 shows distribution of infants involving $56.5 \%$ males and $43.5 \%$ females in infants 7 days to 2 months of age.

Pediatric Review: International Journal of Pediatric Research Available online at: www.medresearch.in 522|P a g e 
Table-3: Sex distribution of study infants (7 days-2 months).

\begin{tabular}{|c|c|c|}
\hline & Number & Percent (\%) \\
\hline Male & 39 & $\mathbf{5 6 . 5 \%}$ \\
\hline Female & $\mathbf{3 0}$ & $\mathbf{4 3 . 5 \%}$ \\
\hline Total & $\mathbf{6 9}$ & $\mathbf{1 0 0} \%$ \\
\hline
\end{tabular}

Seventy-five young infants were recruited from the OPD and Emergency. Table 4 depicts the relationship between recruitment and hospitalization. Total of 73 infants were admitted, 12 from OPD and 61 from Emergency. 77 infants were sent home after initial management in hospital. Total of 73 infants were admitted due to multiple morbidities

Table-4: Relationship between recruitment and hospitalization (0-2 months).

\begin{tabular}{|c|c|c|c|}
\hline & Admitted & Sent Home & Total \\
\hline OPD & 12 & 63 & 75 \\
\hline Emergency & 61 & 14 & 150 \\
\hline Total & 73 & 77 & 150 \\
\hline
\end{tabular}

Out of 150 young infant, 81 infants were 0-7 days old and remaining 69 were in the age group from 7 days upto 2 months and mean age is 13.27 days which is depicted in Table 5.

Table-5: Age distribution of study infants.

\begin{tabular}{|c|c|c|}
\hline & Number & Percent (\%) \\
\hline $0-7$ days & $\mathbf{8 1}$ & $\mathbf{5 4 \%}$ \\
\hline 7 days-2 months & $\mathbf{6 9}$ & $\mathbf{4 6 \%}$ \\
\hline Total & $\mathbf{1 5 0}$ & $\mathbf{1 0 0 \%}$ \\
\hline
\end{tabular}

\section{Utility of algorithm to identify jaundice}

The agreement between the IMNCI algorithm and Gold Standard in identification of jaundice is as followsSevere jaundice was present in 12 infants according to IMNCI and 6 infants according to Gold Standard in 0-2 months of age as depicted in Table 6.

Table-6: Accuracy of algorithm to identify jaundice (0-2 months)

\begin{tabular}{|c|c|c|}
\hline & IMNCI & Gold Standard \\
\hline No Jaundice & 104 & 114 \\
\hline Jaundice & 34 & 60 \\
\hline Severe jaundice & 12 & $\mathbf{1 5 0}$ \\
\hline Total & $\mathbf{1 5 0}$ & 6 \\
\hline
\end{tabular}

The predictive utility of algorithm for the diagnosis of severe jaundice with a sensitivity of $100 \%$ and specificity of $75 \%$ in $0-2$ months of age group as shown in Table 7.

Table-7: Utility to identify severe jaundice (0-2 months).

\begin{tabular}{|c|c|}
\hline Sensitivity & $100 \%$ \\
\hline Specificity & $95 \%$ \\
\hline Positive predictive value & $50 \%$ \\
\hline Negative predictive value & $100 \%$ \\
\hline
\end{tabular}




\section{Original Research Article}

Table 8 and 9 shows accuracy of algorithm and its utility respectively for severe jaundice in infants 0-7 days of age. Severe jaundice was present in 9 infants according to IMNCI and 5 infants according to Gold Standard in 0-7 days of age as depicted in Table 8.

Table-8: Accuracy of algorithm to identify jaundice (0-7 days).

\begin{tabular}{|c|c|c|}
\hline & IMNCI & Gold Standard \\
\hline No Jaundice & 57 & 58 \\
\hline Jaundice & 15 & 5 \\
\hline Severe Jaundice & 9 & $\mathbf{8 1}$ \\
\hline Total & $\mathbf{8 1}$ & 18 \\
\hline
\end{tabular}

The predictive utility of algorithm for the diagnosis of severe jaundice with a sensitivity of $100 \%$ and specificity of $94.7 \%$ in $0-7$ days of age group was found as shown in Table 9.

Table-9: Utility of identity severe jaundice (0-7 days).

\begin{tabular}{|c|c|}
\hline Sensitivity & $100 \%$ \\
\hline Specificity & $94.7 \%$ \\
\hline Positive predictive value & $55.5 \%$ \\
\hline Negative Predictive value & $100 \%$ \\
\hline
\end{tabular}

Table 10 and 11 shows accuracy of algorithm and its utility respectively for severe jaundice in infants 7-59 days of age

Severe jaundice was present in 3 infants according to IMNCI and 1 infant according to Gold Standard in 7-59 days of age as depicted in Table 10 .

Table-10: Accuracy of algorithm to identify jaundice (7-59 days).

\begin{tabular}{|c|c|c|}
\hline & IMNCI & Gold Standard \\
\hline No Jaundice & 47 & 12 \\
\hline Jaundice & 19 & 1 \\
\hline Severe Jaundice & 3 & $\mathbf{6 9}$ \\
\hline Total & $\mathbf{6 9}$ & 12 \\
\hline
\end{tabular}

The predictive utility of algorithm for the diagnosis of severe jaundice with a sensitivity of $100 \%$ and specificity of $97 \%$ in 7-59 days of age group was observed as shown in Table 11.

Table-11: Utility to identify severe jaundice (7-59 days).

\begin{tabular}{|c|c|}
\hline Sensitivity & $100 \%$ \\
\hline Specificity & $97 \%$ \\
\hline Positive predictive value & $33 \%$ \\
\hline Negative Predictive value & $100 \%$ \\
\hline
\end{tabular}

\section{Discussion}

During the mid-1990s, the World Health Organization (WHO), in collaboration with UNICEF and many other agencies, institutions and individuals, responded to this challenge by developing a strategy known as Integrated Management of Childhood Illness (IMCI) [9].
In health facilities, the IMCI strategy promotes the accurate identification of childhood illness (es) in the outpatient settings, ensures, appropriate combined treatment of all major illnesses, strengthens the counselling of caretakers and the provision of 


\section{Original Research Article}

preventive services, and speeds up the referral of severely ill children [10]. This information contributed to the development of the Integrated Management of Childhood Illness (IMCI) algorithms during the mid1990s, which standardized the management of sick young infants at first-level health facilities [11].

A total of 150 infants between $0-2$ months who fulfilled the study criteria were investigated. Of these 81 (54\%) were $0-7$ days and $69(46 \%)$ were 7 days -2 months of age.

Thus, a considerable number of study infants were between 0-7 days of age to allow us to see whether the IMNCI algorithm which has additionally included early neonatal period (0-7 days), can be utilized with equal efficacy in 0-7 days as in 7 days- 2 months age group. About $46 \%$ of study subjects were females and $54 \%$ were males.

\section{Relationship between recruitment site and hospitalization}

Out of 150 young infants, 75 infants were recruited both form OPD and emergency of hospital. Out of 75 infants recruited form OPD $16.0 \%$ were admitted and remaining $84.0 \%$ were sent home after initial evaluation. The corresponding figures form emergency were 81.35 and $18.7 \%$ respectively.

Similar study conducted by Kaur et al [12] which showed that $97 \%$ of infants coming to emergency were admitted and rest sent home as compared to $54 \%$ admission from OPD. Thus, hospitalization rates among those who presented to the emergency were much higher than those who attended OPD suggesting that a significantly higher proportion of severely ill patients reported to emergency.

However, amongst the patients in early neonatal period (0-7 days), $28 \%$ of infants presenting to OPD were hospitalized and $72 \%$ were sent back home. Corresponding figures for infant presenting to emergency was $80.3 \%$ and $19.7 \%$ respectively.

Goswami et al [3] showed that $66 \%$ of infants in early neonatal period (0-7 days) were admitted as compared to $52 \%$ in 7 days -2 months age groups. Gupta et al [6] showed that 595 of infants were admitted and rest $41 \%$ were sent back home in 7 days -2 months age groups. Neonates less than 7 days of age brought to the hospital were sicker and were hospitalized when they presented to OPD and emergency.

\section{Jaundice diagnostic agreement}

Out of 150 infants, according to IMNCI and Gold Standard severe jaundice was present in $12(8 \%)$ and $6(4 \%)$ respectively. Severe Jaundice had high sensitivity of $100 \%$ and specificity of $95 \%$. Study conducted by Kaur et al [12] showed that according to gold standard jaundice was present in 50.6\% in 0-7 days and $17.3 \%$ in 7 to 59 days infants. In study conducted by Bhattacharya et al. according to IMNCI Jaundice and severe jaundice was present in $6 \%$ infants and $1.7 \%$ infants respectively as compared to $34 \%$ and $8 \%$ respectively in the present study. In this study $7.7 \%$ infants had jaundice according to gold standard as compared to $20 \%$ in the present study. Sensitivity and Specificity to identify jaundice were $66.67 \%$ and $99.07 \%$ as compared to $100 \%$ and $95 \%$ in the present study [13].

According to bicentric study in Bolivia conducted by Mazzi et al jaundice was present as per IMNCI in 0-7 days and 7-59 days as $49 \%$ and $32.8 \%$ infants as compared to $29 \%$ and $31 \%$ in the present study respectively. In this study jaundice as per gold standard, present in 0-7 days and 7-59 days infants was 55\% and $38.5 \%$ infants as compared to $28 \%$ and $18.8 \%$ in the present study respectively [14]. This high diagnosis of jaundice according to IMNCI and gold standard was similar to that in the present study.

In study conducted by Thummakomma $\mathrm{M}$ et al jaundice was present according to gold standard in 0-7 days as $54.2 \%$ and $7-59$ days as $45.7 \%$ compared to $28 \%$ and $18.8 \%$ in the present study respectively. According to IMNCI jaundice was present $19.8 \%$ as compared to $30.6 \%$ in the present study. Sensitivity, specificity, Positive predictive value and negative predictive value in study conducted by Thummakomma $\mathrm{M}$ et al and in the present study was $61.3 \%, 95 \%, 81.4 \%, 94.6 \%$ and $100 \%, 95 \%, 50 \%, 100 \%$ respectively which is comparable [15].

\section{Conclusion}

The present study was done to validate the IMNCI algorithm in young infants $0-2$ months of age. Of the total 150 infants studied $81(54 \%)$ were $0-7$ days of age and 69 were 7 days- 2 months of age. Seventy-five infants were recruited both from OPD and emergency. As per Gold Standard 73(48.7\%) were hospitalized and $77(51.3 \%)$ were sent back home and managed on outpatient basis after initial evaluation. IMNCI algorithm performed well in identifying severe jaundice with the sensitivity of $100 \%$ and specificity of $95 \%$ 


\section{Original Research Article}

\section{What this study adds to existing knowledge?}

IMNCI Algorithm performed well in identifying severe jaundice and is important tool to diagnose severe jaundice by health care professional.

\section{Author's Contribution}

Dr. Rishi Bansal: Conceived the idea of study, did data collection, supervised the study, participated in study design, reviewed related literature, write up the manuscript.

Dr. Ram Kshitij Sharma: Data collection, supervised the study, analysis of data and interpretation of results, and write up of manuscript.

Funding: Nil, Conflict of interest: None initiated, Permission from IRB: Yes

\section{References}

1. Integrated Management of Childhood Illness (IMCI) Available at https://www.who.int/ maternal_ child_adolescent/ topics/child/imci/en/

2. Lawn JE, Cousens S, Zupan J; Lancet Neonatal Survival Steering Team. 4 million neonatal deaths: when? Where? Why? Lancet. 2005;365(9462):891-900. doi: 10.1016/S0140-6736(05)71048-5.

3. Goswami V, Dutta AK, Singh V, Chandra J. Evaluation of simple clinical signs of illness in young infants (0-2 months) and its correlation with WHO IMCI algorithm (7 days to 2 months). Indian Pediat. 2006; 43(12):1042-1049.

4. World Health Organization. Integrated management of sick child. Bull WHO 1995;73:735-740.

5. Gove S. Integrated management of childhood illness by outpatient health workers: technical basis and overview. The WHO Working Group on Guidelines for Integrated Management of the Sick Child. Bull World Health Organ. 1997;75(1):7-24.

6. Gupta R, Sachdev HPS, Shah D. Evaluation of the WHO/UNICEF algorithm for integrated management of childhood illness between the age of one week to two months. Indian Pediatr. 2000;37:383-390.
7. Kalter HD, Schillinger JA, Hossain M, Burnham G, Saha S, de Wit V, et al. Identifying sick children requiring referral to hospital in Bangladesh. Bull World Health Organ. 1997;75(1):65-75.

8. What is Integrated Management of Childhood Illness (IMCI)? Available at https://www.who.int/maternal_ child_adolescent/child/imci/background/en/.

9. Madhu Gupta, Arun Kumar Aggarwal. Feasibility study of IMNCI guidelines on effective breastfeeding in a rural area of north India. Ind J Comm Med. 2008;33 (3):201-203. doi: 10.4103/0970-0218.42067.

10. Patwari AK, Raina N. Integrated Management of Childhood Illness (IMCI): a robust strategy. Ind J Pediat. 2002;69(1):41-48. doi: https://doi.org/10. 1007/ BF02723776.

11. Tulloch J. Integrated approach to child health in developing countries. The Lancet. 1999;354:SII16-20. doi: https://doi.org/10.1016/S0140-6736(99)90252-0.

12. Kaur S, Singh V, Dutta AK, Chandra J. Validation of IMNCI algorithm for young infants (0-2 months) in India. Indian Pediatr. 2011;48(12):955-960. Epub 2011 Mar 15. doi: 10.1007/s13312-011-0155-1.

13. Bhattacharyya A, Mukherjee S, Chatterjee C, Dasgupta S. Assessment of validity and reliability of IMNCI algorithm in comparison to provisional diagnosis of senior pediatricians in a tertiary hospital of Kolkata. J Fam Med Primary care. 2013;2(2):173-177. doi: 10.4103/2249-4863.117393.

14. Mazzi E, Bartos AE, Carlin J, Weber MW, Darmstadt GL, Bolivia Clinical Signs Study Group. Clinical signs predicting severe illness in young infants ( $<60$ days) in Bolivia. Journal of tropical pediatrics. 2010 Feb 8;56(5):307-316. doi: https://doi.org/10. 1093/ tropej/fmq005.

15. Thummakomma M, Ramesh P, Reddy M, Balram B. Utility of implementing integrated management of neonatal and childhood illness (IMNCI) algorithm in a tertiary care hospital for the young infants of age 0-2 months. J Evid Med Healthcare. 2016;3(89):4868-4872. doi: $10.18410 / \mathrm{jebmh} / 2016 / 1026$.

\section{How to cite this article?}

Bansal R, Sharma R.K. Validation of WHO-IMNCI algorithm for jaundice in 0-2 months aged infants. Int J Pediatr Res.2019;6(10): 521-526.doi:10.17511/ijpr.2019.i10.05 